EVS28

KINTEX, Korea, May 3-6, 2015

\title{
Economic assessment of strategies to deploy publicly accessible charging infrastructure
}

\author{
Carlos Madina ${ }^{1}$, Heike Barlag ${ }^{2}$, Giovanni Coppola ${ }^{3}$, Ines Gomez ${ }^{1}$, Raul Rodriguez ${ }^{1}$, Eduardo \\ Zabala $^{1}$ \\ ${ }^{I}$ TECNALIA, c/Geldo, Ed.700, Parque Tecnológico de Bizkaia, 48160 Derio, Spain, carlos.madina@tecnalia.com, \\ ${ }^{2}$ Siemens $A G,{ }^{3}$ Enel Distribuzione
}

\begin{abstract}
From the end user perspective, the main barriers for widespread electric vehicle (EV) adoption are high purchase cost and range anxiety, both regarding battery capacity and availability of accessible EV charging infrastructure. Governments and public bodies in general are taking steps towards overcoming these barriers by, among others, setting up regulatory requirements regarding standardisation, customer information and recommending objectives of publicly accessible charging infrastructure. However, the economic performance of publicly accessible charging infrastructure is unknown and any deployment plan should be backed up by a rigorous cost-benefit analysis, to check the efficiency of the plan in economic terms.

This paper presents the results of the economic assessment performed within the FP7 EU-funded Green eMotion project, where relevant conclusions for helping industry strategic approach and decision makers have been taken.
\end{abstract}

Keywords: Business models, Economic assessment, Public access, Charging infrastructure

\section{Introduction}

A business model (BM) describes the rationale of how an organisation creates, delivers and captures value. Hence, a BM looks into many aspects of the business developer and its environment, including customers, offer, infrastructure, and financial viability.

The BM analysis presented in this paper is based on the work performed in Green eMotion project [1]. This BM work seeks to identify the BMs most suited to facilitate large-scale electric vehicles (EV) roll-out in terms of social acceptance, commercial viability and system/environmental impact. More details can be found in [2].
This economic assessment is based on data provided by several Green eMotion partners; vehicle manufacturers (BMW, Nissan, Daimler), electric utilities (RWE, Enel), equipment manufacturers (Siemens, Bosch) and ICT companies (IBM), which ensures the relevance of the data and assumptions considered in the analysis. When needed, the data have been complemented with additional sources of information, either external [3] or internal [4] to the project.

\section{Electric mobility framework}

EV-ecosystem is a network of actors who interrelate with each other and all call for a positive business case. 
Therefore the BM analysis does not only focus on the actors who want to launch the business (e.g. Electric Vehicle Supply Equipment (EVSE) Operator or Electric Vehicle Service Provider (EVSP)), but also on all the players that can be involved across the value chain, including some regulated companies (such as Transmission System Operator (TSO) or Distribution System Operator (DSO)) and liberalized stakeholders (such as electricity retailers or producers).

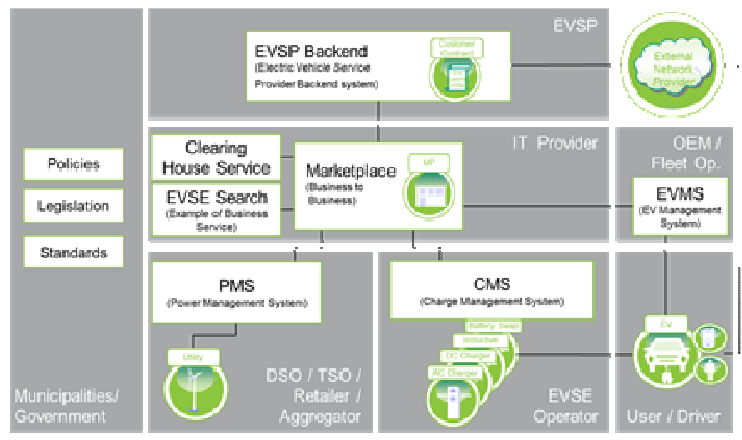

Figure 1: Green eMotion Building Blocks and Swimlanes

The actors in this network may have different roles and responsibilities, and the relationships between them may also be different. Depending on the regulation in each country:

- Roaming agreements between EVSPs and EVSE Operators may be compulsory or optional.

- Charging service may include electricity or not, i.e. EV customers may receive an allinclusive service from a single supplier or they may need to pay for access to the EVSE and for the electricity separately.

Moreover there is a number of alternatives for the characteristics of the EV charging process:

- The place where charging takes place: in publicly accessible EVSE in public domain (curb side) in publicly accessible EVSE in private domain (malls, airports), in restricted-access EVSE in private domain (workplaces) or in private EVSE (home charging).

- The type of charging technology: conductive or inductive, battery swapping.

- The speed of charging: slow (e.g. $3.7 \mathrm{~kW}$ ), semi-fast (e.g. $11 \mathrm{~kW}$ or $22 \mathrm{~kW})$ or fast $(50$ $\mathrm{kW}$ or more).

The contractual relationships between the different parties (in particular, between EVSPs and EVSE Operators) may also be of bilateral nature, or they may subscribe to a marketplace for selling and buying e-mobility services.

\section{EV charging location scenarios}

The large-scale roll-out of EVs will depend on different types of charging infrastructure. Hence, four charging location scenarios are considered:

1. Traffic hotspot charging: Publicly accessible EVSE on private domain and semi-fast charging $(11 \mathrm{~kW}$ or $22 \mathrm{~kW})$. There is no roaming, as the hotspot operator is both the EVSP and the EVSE Operator.

2. Highway charging: Publicly accessible EVSE on private domain and fast charging $(50 \mathrm{~kW})$. There is roaming through the marketplace.

3. Private home charging: Private EVSE and slow charging $(3.7 \mathrm{~kW})$. No roaming required, as the EVSE Operator is the EV customer.

4. Public charging spot for street side parking: Publicly accessible on public domain and semi-fast charging ( $11 \mathrm{~kW}$ or $22 \mathrm{~kW})$. There is roaming through the marketplace.

Only conductive charging is considered, as it is expected to meet most of the charging events, at least in the short to medium term.

\section{Methodology}

In order to assess whether there is room for developing a positive business model for all the actors involved, the analysis assumes that both the EVSE Operator and the EVSP use a pricing strategy which allow them recover their costs. Then, its effect on EV customers is compared against a similar situation for an Internal Combustion engine vehicle (ICV).

\subsection{Main cost components}

\subsubsection{EVSE Operator}

In the most general case, the costs for the EVSE Operator are those related to the charging infrastructure, electricity bill costs, communication costs, costs for accessing the marketplace and staff and overhead costs.

All the issues related to the operation of the EVSE are outsourced to a third party. EVSE Operator is an already existing company, so that staff and overhead costs are assumed to be negligible.

As a result, the costs for the EVSE Operator are some fixed costs per EVSE (amortisation, O\&M, communications and the fixed part of the electricity bill) and some variable costs which depend on each charging session (variable part of the electricity bill).

The electricity bill costs depend on each country. As an example, annual EVSE Operator's in Spain are calculated by means of equation (1): 
$\left\{\left[\int\left[\left(P^{*} T p * 12\right)+\sum_{i=1}^{3}\left(S h_{i} * T e i\right) * E * C\right] *(1+\right.\right.$ Etax $\left.\left.\left.)\right\}+(M * 12)\right) *(1+V A T)\right]+\frac{\text { EVSEinv }}{\frac{(1+7 \%)^{7.5}-1}{7 \% *(1+7 \%)^{7.5}}}+$ EVSEO \& $\left.M\right\} * N+$ MPEVSEO

On the other hand, EVSE Operator's income mainly relies on the EV charging sessions made, although other sources of revenue might also be envisaged, as shown in equation (2).

EVSE Operators' income $=\left(C P^{*} N^{*} C\right)+O S$ The parameters used in equations (1) and (2) are shown in Table 1.

Table 1: Parameters for calculating EVSE Operator's cost and incomes

\begin{tabular}{|c|c|c|}
\hline Parameter & Description & Units \\
\hline $\mathrm{P}$ & Power & $\mathrm{kW}$ \\
\hline $\mathrm{Tp}$ & $\begin{array}{l}\text { Power component of } \\
\text { the electricity price }\end{array}$ & $€ / \mathrm{kW} / \mathrm{month}$ \\
\hline $\mathrm{Sh}_{\mathrm{i}}$ & $\begin{array}{l}\text { Share of charges in } \\
\text { each period } \mathrm{i}\end{array}$ & $\%$ \\
\hline $\mathrm{Te}_{\mathrm{i}}$ & $\begin{array}{l}\text { Energy component of } \\
\text { the electricity price }\end{array}$ & $€ / \mathrm{kWh}$ \\
\hline $\mathrm{E}$ & $\begin{array}{l}\text { Energy charged per } \\
\text { session }\end{array}$ & $\mathrm{kWh}$ \\
\hline $\mathrm{C}$ & $\begin{array}{l}\text { Number of charging } \\
\text { session }\end{array}$ & Sessions/year \\
\hline $\mathrm{M}$ & Meter renting & $€ /$ month \\
\hline Etax & Electricity tax & $\%$ \\
\hline VAT & Value added tax & $\%$ \\
\hline $\mathrm{EVSE}_{\text {inv }}$ & EVSE investment & $€$ \\
\hline $\mathrm{EVSE}_{\mathrm{O} \& \mathrm{M}}$ & EVSE O\&M costs & $€ /$ EVSE/year \\
\hline $\mathrm{N}$ & Number of EVSEs & EVSE \\
\hline $\mathrm{MP}_{\text {EVSEO }}$ & Marketplace access & $€ /$ year \\
\hline $\mathrm{CP}$ & Charging service price & $€ /$ session \\
\hline OS & $\begin{array}{l}\text { Other sources of } \\
\text { revenue }\end{array}$ & $€ /$ year \\
\hline
\end{tabular}

\subsubsection{EVSP}

The EVSP has some fixed costs and some variable costs. Fixed costs include costs for accessing the marketplace, communication costs, staff and overhead costs and radio-frequency identification (RFID) card costs. It is assumed that they are recovered by means of an annual subscription fee of $120 € /$ year (details can be found in [2]) to be paid by EV customers who, in return, receive the right to charge in the highway charging and in the public charging spot for street side parking scenarios. Variable costs are passed through directly to EV customers, who must pay the value added tax (VAT) and therefore the EV charging price they will pay is slightly higher than charging service price requested by the EVSE Operator.

\subsubsection{EV customers}

EV customers also have some fixed and some variable costs which depend on vehicle usage. In order to compare the total cost of ownership (TCO) for an EV and for an equivalent ICV, the same annual mileage and investment decisions (expected vehicle lifetime and discount rate) are considered.

In the most general case, fixed costs include the vehicle amortisation cost, EVSP subscription price and the EVSE amortisation cost and annual EVSE O\&M costs (only relevant in the case of private home charging scenario). Variable costs include fuel, O\&M and the EV charging. The EV charging cost in each scenario is calculated by multiplying the number of times that EV customers use each scenario per year by the EV charging price.

Considering a discount rate of $7 \%$, a vehicle lifetime of 12 years, the O\&M costs $(1.1 € / 100 \mathrm{~km}$, $3.3 € / 100 \mathrm{~km}$ and $3.6 € / 100 \mathrm{~km}$ for $\mathrm{EVs}$, gasoline and diesel ICVs respectively [4]) and a lifetime of 7.5 years for the EVSE (when applicable), the TCO for each vehicle can be calculated by using equations (3), (4) and (5), whose parameters are described in Table 2:

Table 2: Parameters for calculating TCOs

\begin{tabular}{|l|l|l|}
\hline Parameter & Description & Units \\
\hline $\mathrm{K}$ & Annual mileage & $\mathrm{km} /$ year \\
\hline $\mathrm{U}_{\mathrm{i}}$ & $\begin{array}{l}\text { Number of charging } \\
\text { sessions in each } \\
\text { scenario i (per EV) }\end{array}$ & Sessions/year \\
\hline $\mathrm{FC}$ & Fuel consumption & $1 / \mathrm{km}$ \\
\hline $\mathrm{FP}$ & Fuel price & $€ / 1$ \\
\hline $\mathrm{S}$ & $\begin{array}{l}\text { EVSP subscription } \\
\text { price }\end{array}$ & $€ /$ year \\
\hline $\mathrm{OM}$ & $\begin{array}{l}\text { Operation \& } \\
\text { Maintenance costs }\end{array}$ & $€ /$ year \\
\hline
\end{tabular}

$\mathrm{TCO}_{\mathrm{EV}}=$

$\frac{\text { Vehicle price }_{\mathrm{EV}}}{\frac{(1+7 \%)^{12}-1}{7 \% *(1+7 \%)^{12}}}+S+\frac{\text { EVSE }_{i n v}}{\frac{(1+7 \%)^{7.5}-1}{7 \% *(1+7 \%)^{7.5}}}+E V S E_{O \& M}$

$+(O M * K)+E V$ charging cost

$\mathrm{TCO}_{\text {Gasoline }}=$

$\frac{\text { Vehicle price }_{\text {Gasoline }}}{\text { enc }}+(F C * F * K)+(O M * K)$

$\frac{(1+7 \%)^{12}-1}{7 \% *(1+7 \%)^{12}}$ 


$$
\begin{aligned}
& \mathrm{TCO}_{\text {Diesel }}= \\
& \frac{\text { Vehicle price }_{\text {Diesel }}}{(1+7 \%)^{12}-1} \\
& \frac{\left(F C^{*} F P^{*} K\right)+(O M * K)}{7 \%}
\end{aligned}
$$

The traffic hotspot charging scenario and the highway charging scenario will be used quite seldom by EV customers and, hence, are not expected to have a big impact on their TCO. Therefore, only the variable costs of EV customers are considered and the relative cost for EV customers of the charging event must be compared against refuelling cost on equivalent trip with an ICV. In the cases EVs are competitive with gasoline (6) and/or diesel (7) the charging price $(€ / \mathrm{km})$ meets the following conditions:

$$
\begin{aligned}
& \mathrm{CP}_{\mathrm{i}} \leq\left(\frac{5.6 * \mathrm{FP}_{\text {Gasoline }}}{100}+0.022\right) * \frac{E_{i}}{E F_{i} *(1+V A T)} \\
& \mathrm{CP}_{\mathrm{i}} \leq\left(\frac{4.9 * \mathrm{FP}_{\text {Diesel }}}{100}+0.025\right) * \frac{E_{i}}{E F_{i} *(1+V A T)}
\end{aligned}
$$

On the contrary, private home charging and public spot for street side parking are the most used scenarios and, as a result, the impact of these scenarios in EV customer's TCO is higher and, thus, also fixed costs are included in the analysis.

Vehicles prices and fuel prices are different across Europe. The prices for Spain (base case), Germany and the Netherlands are considered.

\subsection{Usage patterns}

Although the pace of electric mobility adoption is not as fast as expected at the beginning of the decade, the strong commitment by public bodies, electric utilities and original equipment manufacturers (OEMs) is slowly setting the conditions for a more massive adoption of EVs. The BMs presented here can only be profitable if there is a big enough EV market behind them. Therefore, the situation described in this paper (having the EVSE used at least once per day) takes into account such market, although it may not happen until 2 or 3 years from now.

\section{Traffic hotspot charging}

This scenario is used by EV customers when they charge at a publicly accessible EVSE on private domain and which is located in a point of interest (POI) and it is assumed that there is an already existing direct payment system.
As a result, the POI operator is at the same time the EVSE Operator and the EVSP (no roaming required and, thus, no marketplace). The charging speed is assumed to be semi-fast $(22 \mathrm{~kW})$ and the EVSE is assumed to have two outlets.

\subsection{Base case}

In this scenario, the relative cost for EV customers of the charging event must be compared against refuelling cost of an equivalent trip with an ICV and, hence, equations (6) and (7) are used.

The different parameters used are shown below.

Table 3: Parameters for the Traffic Hotspot scenario (Spain)

\begin{tabular}{|c|c|c|}
\hline Parameter & Value & Unit \\
\hline $\mathrm{P}$ & $2 * 22$ & $\mathrm{~kW}$ \\
\hline $\mathrm{E}$ & 10 & $\mathrm{kWh}$ \\
\hline $\mathrm{EF}$ & 120 & $\mathrm{Wh} / \mathrm{km}$ \\
\hline $\mathrm{N}$ & 1 & $\mathrm{EVSE}$ \\
\hline VAT EVSE op & 0 & $\%$ \\
\hline VAT EV user & 21 & $\%$ \\
\hline $\mathrm{MP}_{\mathrm{EVSEO}}$ & 0 & $€ /$ year \\
\hline $\mathrm{Tp}$ & 6.832399 & $€ / \mathrm{kW} / \mathrm{month}$ \\
\hline $\mathrm{Te}_{1}$ & 0.122383 & $€ / \mathrm{kWh}$ \\
\hline $\mathrm{Te}_{2}$ & 0.096216 & $€ / \mathrm{kWh}$ \\
\hline $\mathrm{Te}_{3}$ & 0.065923 & $€ / \mathrm{kWh}$ \\
\hline $\mathrm{M}_{2}$ & 1.36 & $€ / \mathrm{month}$ \\
\hline $\mathrm{EVSE}_{\text {Inv }}$ & 10500 & $€ / \mathrm{EVSE}$ \\
\hline $\mathrm{EVSE}_{\mathrm{O} \& \mathrm{M}}$ & 1725 & $€ / \mathrm{EVSE} / \mathrm{year}$ \\
\hline
\end{tabular}

The maximum charging service price to be requested by the EVSE Operator so that EV customers' mileage cost is competitive against ICVs is calculated by using equations (6) and (7): $7.06 €$ (gasoline) and $6.21 €$ (diesel) per session. The prices for EV customers (including VAT) are thus 8.54 and $7.52 € /$ session, respectively.

The costs and incomes for the EVSE Operator are obtained by using equations (1) and (2). When no additional sources of income are considered, the minimum number of charging sessions per EVSE to have a positive result (income > cost) can be calculated by equations (8) and (9) when compared against gasoline and diesel, respectively:

$$
\begin{aligned}
& 7.0611 * \mathrm{C}=7380.16+1.0396 * \mathrm{C} \\
& 6.2134 * \mathrm{C}=7380.16+1.0396 * \mathrm{C}
\end{aligned}
$$

This means that, if each traffic hotspot EVSE is used to charge more than 3.91 (3.36) times per day on average, i.e. 1.96 (1.68) charging sessions per outlet and per day, there is room for a pricing strategy that allows the EVSE Operator recover its costs, while making EV customers' mileage cost competitive against diesel (gasoline) ICVs. 
If there are enough EVs on the road, these usage rates are likely to be achieved in traffic hotspots. The EVSE Operator can also offer competitive prices to EV customers at lower EVSE usage rates if other sources of revenue are found. One of the best alternatives is to use the EVSE for advertisement purposes. Considering the shape of different advertising alternatives, prices for phone kiosks are considered. Depending on the location and the actual advertising surface, its price ranges between $4000 €$ and $10000 €$. Incomes in the upper range (10 $000 € /$ year) are enough to pay for all the costs of the EVSE, even if it is never used. In the lower range (4000 $€$ /year), the required EVSE usage to be able to offer competitive prices is reduced to 1.54 (gasoline) and 1.79 (diesel) sessions/day, which is even more likely to happen in traffic hotspots.

\subsection{Sensitivity analysis}

\subsubsection{Battery size}

The battery size considered in the base case is based on existing EVs, but it is expected to increase in the future to allow EVs drive a longer range. With bigger batteries, EV customers will charge more energy per session, but they will also charge less often. If, for example, an average of $20 \mathrm{kWh}$ per charging session is assumed, the maximum charging price doubles and the required EVSE usage rate is halved.

The impact on the BM depends on whether EV customers with bigger batteries use hotspot EVSEs more often (as they gain confidence in EV use and, thus, more EVs are sold and they are used more frequently) or more seldom (even if the EV market and usage increases, most EV customers still use private home charging.

\subsubsection{EV efficiency}

The efficiency considered in the base case is in the lower range of existing EVs. If it is assumed to be in the upper range, $(150 \mathrm{Wh} / \mathrm{km})$, the driving range with the same $10 \mathrm{kWh}$ charging session will be reduced. Under these conditions, more than 5 charging sessions per EVSE and per day are required for the EVSE Operator to offer competitive prices to $\mathrm{EV}$ customers.

As for the battery size, two opposite effects happen at the same time; a lower driving range results in a higher mileage cost and, thus, the price per charging session needs to be lower to compete with ICVs; while, on the other hand, as driving range decreases, EV customers need to charge more often, increasing EVSE usage rate.

\subsubsection{Germany}

The values used for the analysis in Germany are the same as for Spain, except for the electricity bill (whose structure and prices are different) and the fossil fuel prices.

The maximum charging price to be requested by a German traffic hotspot EVSE Operator, so that EV customers have a mileage cost which is competitive against ICVs, is $9.23 €$ (gasoline) and $7.73 €$ (diesel) per charging session, 19\% VAT included.

Considering the costs and incomes for a traffic hotspot EVSE Operator in Germany, if each EVSE is used to charge more than $1.26(0.96)$ charging sessions per outlet and per day, there is room for a pricing strategy that allows the EVSE Operator recover its costs and still offer a competitive mileage cost for EV customers in comparison with diesel (gasoline) ICVs.

Therefore, the required usage rate in Germany is lower than in Spain.

\subsubsection{The Netherlands}

As in the case of Germany, the differences with the base case appear in the electricity bill structure and prices, as well as in fossil fuel prices.

The maximum EV charging prices for EV customers to have a competitive mileage cost with gasoline and diesel are $10.01 €$ and $7.93 €$ per charging session, respectively (including 21\% VAT).

If each EVSE installed is used to charge more than 1.58 (1.15) charging sessions per outlet and per day there is room for a pricing strategy that allows the EVSE Operator recover its costs and still offer a competitive mileage cost for EV customers in comparison with diesel (gasoline) ICVs.

These target usage rates are slightly lower than in Spain.

\section{Highway charging}

Under this scenario, EV customers charge at a publicly accessible EVSE on private domain and which is located in a highway, most likely in an existing fuel filling station. The aim of this EVSE is to allow EV customers have a fast $(50 \mathrm{~kW})$ charging session, so that they can continue their trip. EV customers are assumed to use the roaming agreement of their EVSP with the highway EVSE Operator (through the marketplace) to be able to charge their EVs. 


\subsection{Base case}

As in the traffic hotspot scenario, the relative cost for EV customers of each highway charging event must be compared against refuelling cost of an equivalent trip with an ICV.

Table 4: Parameters for the Highway scenario (Spain)

\begin{tabular}{|c|c|c|}
\hline Parameter & Value & Unit \\
\hline $\mathrm{P}$ & 50 & $\mathrm{~kW}$ \\
\hline $\mathrm{E}$ & 12 & $\mathrm{kWh}$ \\
\hline $\mathrm{EF}$ & 150 & $\mathrm{Wh} / \mathrm{km}$ \\
\hline $\mathrm{N}$ & 1 & $\mathrm{EVSE}$ \\
\hline VAT EVSE op & 0 & $\%$ \\
\hline VAT EV user & 21 & $\%$ \\
\hline $\mathrm{MP}_{\text {EVSEO }}$ & 2000 & $€ /$ year \\
\hline $\mathrm{Tp}$ & 6.832399 & $€ / \mathrm{kW} / \mathrm{month}$ \\
\hline $\mathrm{Te}_{1}$ & 0.122383 & $€ / \mathrm{kWh}$ \\
\hline $\mathrm{Te}_{2}$ & 0.096216 & $€ / \mathrm{kWh}$ \\
\hline $\mathrm{Te}_{3}$ & 0.065923 & $€ / \mathrm{kWh}$ \\
\hline $\mathrm{M}$ & 1.36 & $€ / \mathrm{month}$ \\
\hline $\mathrm{EVSE}_{\text {Inv }}$ & 27150 & $€ /$ EVSE \\
\hline $\mathrm{EVSE}_{\mathrm{O} \& \mathrm{M}}$ & 3075 & $€ / \mathrm{EVSE} /$ year \\
\hline
\end{tabular}

The maximum charging service price to be requested by the EVSE Operator so that EV customers have a mileage cost which is competitive against ICVs is calculated by using equations (6) and (7) and the results are $8.20 €$ and $7.22 €$ per charging session, gasoline and diesel respectively (21\% VAT included).

The costs and incomes for the EVSE Operator are obtained by using equations (1) and (2) and the obtained results show that the highway EVSE needs to be used to charge more than 8.23 (7.02) times per day on average to be competitive with diesel (gasoline). This target usage seems to be quite difficult to reach in a highway EVSE.

Therefore, the EVSE Operator needs to look for additional sources of revenue. The impact of the advertisement, although it is a good option, is expected to be lower than in the traffic hotspot.

If the expected income is in the lower range of the advertisement options for phone kiosks (about $4000 € /$ year) the required EVSE usage would be 5.04 sessions/day (gasoline) and 5.91 (diesel).

Since charging time is about 15 minutes, another good alternative for the highway charging case is the attraction of clients to an existing shop or restaurant. Assuming a 30\% profit rate, if each EV customer spends $5 \mathrm{€} / \mathrm{session}$, the extra income for the EVSE Operator is $1.5 € /$ session. In this case, the numbers of sessions to be competitive with diesel are:
- Ads $=0 \rightarrow 6.25$ sessions/day.

- Ads $=4000 € /$ year $\rightarrow 4.48$ sessions/day.

- Ads $=10000 € /$ year $\rightarrow 1.84$ sessions/day.

As a result, the highway EVSE Operator needs to obtain a good advertising contract and to attract EV consumers to other businesses (shop or restaurant) to be able to offer prices that make electric mobility competitive with traditional ICVs. An additional constraint is that highway charging competes with private home charging, at least in the cases where EV customers can charge their $\mathrm{EV}$ s at home.

Therefore, the profitability of highway charging scenario is not as clear as the one in the traffic hotspot case.

\subsection{Sensitivity analysis}

\subsubsection{EVSE amortisation cost}

Future prices of EVSE are expected to decline. Likewise, future EVSE is expected to last longer.

Therefore, under the assumptions of a $10 \%$ reduction in hardware costs (2000 €) and a lifetime extension to 10 years, the annual amortisation cost is about $1200 € /$ EVSE lower than in the base case, so the required EVSE usage to offer competitive prices to EV customers (against diesel ICVs) is also reduced to about 7.5 charging sessions per day, which is still too high, so the extra incomes (advertising, shop) are still required.

\subsubsection{Battery size}

As discussed in the traffic hotspot case, the battery size affects the amount of energy to be charged per session. Assuming again that the amount of energy to be charged per session is doubled $(24 \mathrm{kWh})$, the maximum charging price that the EVSE Operator can ask so that EV customers have a competitive mileage cost with ICVs is also doubled.

The required amount of charging sessions for EVSE Operators to offer competitive prices to EV customers is calculated for different incomes from advertisement (A) and shop/restaurant (S) and presented in Table 5.

Table 5: Highway EVSE usage requirements for different co-financing alternatives (bigger battery size)

\begin{tabular}{|c|c|c|}
\cline { 2 - 3 } \multicolumn{1}{c|}{} & $\begin{array}{c}\mathrm{S}=0 \\
\text { €/session }\end{array}$ & $\begin{array}{c}\mathrm{S}=1.5 \\
\text { €/session }\end{array}$ \\
\hline $\mathrm{A}=0$ €/year & 4.12 & 3.55 \\
\hline $\mathrm{A}=4000 € /$ year & 2.95 & 2.55 \\
\hline $\mathrm{A}=10000$ €/year & 1.21 & 1.05 \\
\hline
\end{tabular}


An increase in EV battery size is expected to have a positive impact in the highway charging scenario as the EV market is expected to grow, together with EV customers' confidence to make longer trips and, hence, use highway charging more often. However, unless charging power is also increased (which would lead to higher electricity bill and EVSE costs), the charging process would almost last for half an hour.

Therefore, a valet service seems to be required, in order to allow for several charging sessions per day.

\subsubsection{EV efficiency}

EV efficiency is not expected to be better than the one in the base case. Quite on the contrary, it is likely that existing EVs have lower efficiencies while driving at highway speed.

If efficiency is considered to be $180 \mathrm{Wh} / \mathrm{km}, \mathrm{EV}$ mileage cost rises (shorter distance travel with the same expenditure) and, thus, it needs lower EV charging prices (4.97 €/session) to be competitive against ICV (diesel). As a result, EVSE usage rate must increase to about 10.43 sessions per day.

Moreover, this lower EV efficiency may become an additional annoyance for EV customers. However, if battery sizes increase, it is likely that EVs are used more often for highway travelling. Considering a charging event of about 30 minutes, 1.5 people per vehicle and a meal of about $10 € /$ person the benefit would be $4.5 € /$ charging session ( $30 \%$ of expenditure). If an advertising contract of $4000 € / E V S E$ per year is added, competitive prices can be offered to EV customers with 3.39 sessions/day.

\subsubsection{Germany}

Again, the values used for the analysis in Germany are the same as for Spain, except for the electricity bill (whose structure and prices are different) and the fossil fuel prices.

The maximum charging service price to be requested by a German highway EVSE Operator so that $\mathrm{EV}$ customers have a mileage cost which is competitive against gasoline ICV is $8.87 €$ and 7.43 against diesel (19\% VAT included).

Without additional sources of income, the minimum number of charging sessions per EVSE to have a positive result (income > cost) are 8.42 (6.13) times per day on average, in comparison with diesel (gasoline) ICVs. This target usage seems to be quite difficult to reach in a highway EVSE.
As in the case of Spain, the EVSE Operator needs to look for additional sources of revenue. In the lower range of advertising prices (4000 €/year per EVSE), the required EVSE usage to offer competitive prices to EV customers can be reduced to more likely values when there are enough EVs on the road, 3.67 sessions/day (gasoline) and 5.03 sessions/day (diesel). Moreover, the shop and restaurant business in Germany seems to be a growing market. This extra income reduces to the required EVSE usage to even more modest usage rates (2.4-3.3 sessions/day to have prices competitive against gasoline and diesel), even without advertising.

\subsubsection{The Netherlands}

In The Netherlands too, differences appear in the electricity bill structure and prices, as well as in fossil fuel prices. The maximum charging service price to be requested by the highway EVSE Operator so that EV customers have a mileage cost which is competitive against gasoline ICV is 9.61 $€$ and $7.62 €$ against diesel (21\% VAT included). If no additional sources of income are considered, the minimum number of charging sessions per EVSE to have a positive result are 7.88 (5.60) times per day, in comparison with diesel (gasoline) ICVs.

This target usage seems to be quite difficult to reach in a highway EVSE. Looking for additional sources of revenue, advertising reduces the required EVSE usage (3.7-5.2 sessions/day), but it may still be a bit high. Including additional incomes from a shop or a restaurant the usage can be reduced to more likely values when there are enough EVs on the road: 3.78 sessions per day.

\subsubsection{Number of EVSE}

In the base case, it is assumed that the EVSE Operator has a single EVSE. As a result, it must recover the whole costs of being connected to the marketplace. Assuming that the EVSE Operator has more than one filling station, with more than 15 EVSE in total, the fixed costs per can be reduced, also reducing the required EVSE usage to 7.13 sessions/day. Although this EVSE usage is still quite high, there is a clear portfolio effect, as the complete removal of the marketplace access costs would only further decrease the required EVSE usage to 7.07 charging sessions per day.

\subsubsection{Dual charging}

There is already some equipment in the market which allows for dual charging. 
Assuming that its costs are the costs for a fast EVSE $(50 \mathrm{~kW})$ increased by the hardware and maintenance costs of a semi-fast EVSE (22kW) and increasing the required contracted power in the electricity bill, the number of charging sessions to cover EVSE Operator's costs and offer a competitive price to EV customers are 8.35 (gasoline) and 9.79 sessions/day (diesel). Even if this usage rate is higher than in the base case, two charging sessions can take place at the same time, so the required usage per EVSE outlet is almost halved.

The required usage rate can be further reduced to 2.46 sessions per day, if the following assumptions are made:

- Advertising: $4000 € /$ per EVSE and per year.

- Restaurant: $3 € /$ charging session.

- Shop: $0.3726 € /$ charging session.

- Bigger battery: $24 \mathrm{kWh}$ charged per session In an area with enough EVs and with the battery technology allowing longer driving ranges, having two fast charging sessions per day and one semi-fast charging session every two days on average seems much more likely to happen in highway EVSEs than the required usage in the base case.

\section{Private home charging}

This scenario presents the case of charging at a private EVSE. The charging speed is assumed to be slow $(3.7 \mathrm{~kW})$. EV customers themselves are assumed to buy the EVSE required for charging their EVs (so they act as EVSE Operator) and buy electricity through a new supply contract. As a result, there is no need for a marketplace in this scenario. Table 6 presents the different parameters to be used in this scenario.

Table 6: Parameters for the Private Home charging scenario (Spain)

\begin{tabular}{|c|c|c|}
\hline Parameter & Value & Unit \\
\hline $\mathrm{P}$ & 4.6 & $\mathrm{~kW}$ \\
\hline $\mathrm{E}$ & 10 & $\mathrm{kWh}$ \\
\hline $\mathrm{EF}$ & 120 & $\mathrm{Wh} / \mathrm{km}$ \\
\hline $\mathrm{K}$ & 19000 & $\mathrm{~km} /$ year \\
\hline $\mathrm{VAT}$ & 21 & $\%$ \\
\hline $\mathrm{MP}_{\text {EVSEO }}$ & 0 & $€ /$ year \\
\hline $\mathrm{Tp}$ & 3.503619 & $€ / \mathrm{kW} / \mathrm{month}$ \\
\hline $\mathrm{Te}$ & 0.044146 & $€ / \mathrm{kWh}$ \\
\hline $\mathrm{M}$ & 0.81 & $€ /$ month \\
\hline $\mathrm{EVSE}_{\text {Inv }}$ & 1900 & $€ /$ EVSE \\
\hline $\mathrm{EVSE}_{\text {O\&M }}$ & 50 & $€ /$ EVSE/year \\
\hline
\end{tabular}

On the contrary to the two scenarios discussed so far, private home charging scenario is expected to be the preferred charging scenario by those EV customers who can charge their EVs at home. As a result, the impact of this scenario in EV customers' TCO is higher and, thus, both fixed and variable costs must be included in the analysis, so equations (3), (4) and (5) are used for each type of vehicle.

Table 7: TCOs for different type of vehicles

\begin{tabular}{|c|c|}
\cline { 2 - 2 } \multicolumn{1}{c|}{} & TCO $(€ /$ year $)$ \\
\hline Gasoline & 3668.03 \\
\hline Diesel & 3661.16 \\
\hline EV & $2791.20+$ EV charging cost \\
\hline
\end{tabular}

If EV customers charge at home one out of two days $(\mathrm{C}=180$ sessions/year), the annual electricity bill is $358.81 € /$ year and they can drive 15000 $\mathrm{km} /$ year, so $4000 \mathrm{~km}$ must be driven by charging outside home. According to equations (6) and (7) the maximum charging service price that EVSE Operators can ask so that the relative cost of the charging event for EV customers is comparable to the refuelling cost of an equivalent trip with an ICV is 7.06 (gasoline) and $6.21 € /$ session (diesel).

By taking the cost per $\mathrm{km}$ in each case, EV customers' TCO becomes about $3489 € /$ year, which is about $175 € /$ lower than for ICVs.

This means that, under the assumptions described, EV ownership is cheaper than owning an ICV.

\subsection{Sensitivity analysis}

\subsubsection{EVSE investment cost}

The EVSE investment cost considered in the base case is lower than the one proposed in [3] because the technical characteristics may not be needed by EV customers.

If such technical requirements are established by national regulation the increase in EVSE investment cost would not affect the advantages of EVs over ICVs, as the difference is about 105.54 $€$ /year higher, which is lower than the TCO difference. On the contrary, much higher O\&M costs (1175 €/year vs. 50 €/year) due to communication capabilities do change the situation and EV ownership become more expensive than ICV ownership. Therefore, regulators must weigh the advantages of imposing more strict technical conditions for private home charging against the additional costs for EV customers. 


\subsubsection{Annual mileage}

The annual mileage considered in the base case is based on the reported mileage by Nissan Leaf users in Spain, but the average mileage of Spanish drivers is significantly lower (9928 $\mathrm{km} /$ year). Assuming the same distribution of charges as in the base case $(80 \%$ of mileage is based on private home charging, while publicly accessible EVSE are used to cover the remaining $20 \%$ of mileage), and $10000 \mathrm{~km} /$ year, the number of private home charging events is reduced to 95 sessions/year. This number of sessions results in an annual mileage of $8000 \mathrm{~km}$ driven due to private home charging (311.08 $€ /$ year of electricity bill) and the remaining 2000 $\mathrm{km}$ must be driven by charging outside home. The new values for the TCOs are 2646.28 €/year (gasoline), $2750.19 € /$ year (diesel). Even if charging outside home were for free, the TCO for EVs would become 3003.28 €/year (EV), which is more expensive than ICV ownership.

Nevertheless, the average mileage of new cars (less than 5 years old) is almost as high as the one assumed in the base case, and, such analysis seems to better suit the case of electric mobility.

\subsubsection{EV efficiency}

If EV efficiency is assumed to be $150 \mathrm{Wh} / \mathrm{km}$, with the same annual mileage as in the base case (19000 km/year), the required electricity amount is $2850 \mathrm{kWh} /$ year. Assuming that EV customers charge at home one out of two days, the amount of kilometres driven from home charging would be $12000 \mathrm{~km}$ per year and the remaining 7000 $\mathrm{km}$ need to be charged outside home. The new value for the EV TCO is $3743 €$ /year, which is about $80 €$ more expensive than ICV's TCO.

On the contrary, considering a proportion of home charging of $80 \%$, EV customers will charge $2280 \mathrm{kWh} /$ year $(80 \% * 2850 \mathrm{kWh} /$ year $)$ at home and their TCO would be $3498.94 € /$ year, i.e. lower than ICVs'.

\subsubsection{EV purchase cost}

In order to have a comparable TCO (keeping the rest of the assumptions as in the base case), the add-on cost for EV purchase should not exceed $5870 €$ compared to gasoline ICV. Although many other implications are taken into account when designing the subsidy policy, public bodies must ensure that any subsidy must be efficient, in terms of costs, but also in terms of reaching the targets of the policy.

\subsubsection{EV charging included in regular home electricity bill}

Under the assumptions considered for the analysis, the electricity bill in case EV charging is included in the regular home electricity supply contract is about $60 €$ cheaper than in the base case, as long as the contracted power (connection capacity) does not need to be increased.

\subsubsection{Germany}

The differences in electricity bill (structure and prices) and the fossil fuel prices, together with a lower average distance driven per year (15000 $\mathrm{km} /$ year), make TCOs 3483 €/year (gasoline), $3515 €$ /year (diesel) and $4369 € /$ year (EV).

The lack of subsidies for direct EV purchase, together with the lower average annual mileage considered in Germany, make EV ownership much more expensive than ICV's. In order to have the same TCO as gasoline, the EV cannot be more than about 5000-5200 € more expensive than the equivalent gasoline ICV under the assumptions made.

\subsubsection{The Netherlands}

Dutch drivers have an average annual mileage of about $9200 \mathrm{~km}$ which is very close to the average mileage in Spain. Therefore, it can be assumed that EV customers will drive $19000 \mathrm{~km} / \mathrm{year}$ in the Netherlands, as they do in Spain, so the differences only appear in fossil fuel prices and electricity bill (structure and prices). In this case, TCOs become $4525 € /$ year (gasoline), $4353 € /$ year (diesel) and $4853 €$ €year (EV).

Again, the lack of subsidies for direct EV purchase makes EVs more expensive than ICVs. For the considered values, the EV cannot be more than about $6570 €$ more expensive than the equivalent gasoline ICV to have the same TCO.

\section{Public charging spot for street side parking}

This scenario presents the case of charging at a publicly accessible EVSE on public domain. The charging speed is assumed to be semi-fast $(22 \mathrm{~kW})$ and the EVSE is assumed to have two outlets. EV customers are assumed to use the roaming agreement of their EVSP with the public EVSE Operator (through the marketplace) to be able to charge their EVs.

In this scenario, the EVSE will have two different usage patterns and the price to be requested to each of them will be different: 
1. EV customers charging their EVs during the day. The maximum EV charging price to be comparable to diesel ICV is $7.52 € /$ charge (VAT included).

2. EV customers who do not have access to private home charging for overnight charging. The expected number of average charging sessions overnight will not be more than two (number of outlets/EVSE).

In this scenario the impact on the EV customer's TCO will be significant. The TCOs for each type of vehicle are calculated, leading to $3668 € /$ year (gasoline), $3661 € /$ year (diesel) and $2407 € /$ year, plus EV charging costs for EVs. Consequently, EV TCO is lower than ICV ownership as long as charging cost is lower than $1254 €$ /year.

Assuming that EV customers charge 180 times overnight (as assumed in the private home charging scenario), they must drive $4000 \mathrm{~km}$ by using other scenarios (traffic hotspot or highway charging), whose charging prices are set to have mileage costs competitive against ICVs. The worst case for EV customers is when EV charging prices are set to be competitive against gasoline (more expensive), leading to an EV charging cost of about $339 € /$ year. Hence, the cost of public charging in street side parking for EV customers must not exceed 915 €/year, which, for 180 charging sessions, results in 5.085 $€ /$ session $(6.15 € /$ session, including VAT).

Taking these charging service prices into account, the EVSE must be used at least 5.40 times per day on average, which is more than the assumption of using each outlet twice per day, once for daytime and another one for overnight charging.

Therefore, additional sources of income are required. If the lower range of advertising income is considered (4000 €/year), EVSE usage requirements can be reduced to about 3 times per day, i.e. three daytime charging sessions and three overnight charging sessions per outlet every four days, which still seems to be a bit high in the early stages of electric mobility deployment.

\section{Conclusions}

Electric mobility is a complex ecosystem, where different actors are part of a network full of interrelations and all call for a positive business case. Moreover, as it is almost a greenfield land, regulatory and market structures still need to be defined. What is more, despite the advances in the last couple of years, EV market is still incipient, so it is difficult to have profitable business models under present conditions.
The analysis presented in this paper looks a bit ahead in the future (2-3 years), so that the number of EVs on the road is enough to allow for a visible demand of public infrastructure. Four charging location scenarios are presented to describe different charging alternatives for EV customers.

The traffic hotspot scenario can be profitable if 3-4 charging sessions per EVSE and day are achieved, which may be feasible if there are enough EVs on the road due to the hotspot nature of the location. In general, the electricity bill design is an important parameter, with better conditions if fixed costs are low in comparison with variable costs. As an additional source of value to increase EVSE profitability and improve BM performance at lower usage rates, advertising can be an option.

In the highway charging scenario it seems more difficult to make a profit, since 7 charging sessions per EVSE/day are needed. It is likely that the technological development (battery size, efficiency) has a positive effect as EV customers gain confidence and dare to make more longdistance trips. Although advertising does not seem as attractive as in traffic hotspots, it is still a good option to increase EVSE incomes. In highways, however, a shop/restaurant attached to the EVSE could provide extra benefits if EV customers use them while charging.

Private home charging scenario will be the preferred option by EV customers who can charge at home. Under the assumptions taken in this paper, it offers better TCO than ICVs today, but subsidies for EV purchase and preferential use of home charging are required for it.

In the public charging spot for street side parking, more than 4 sessions per EVSE and per day are needed for the EVSE Operator to make a profit while offering competitive prices to EV customers. In this case too, advertising is a good option.

On the grounds of these findings, it seems that EV customers with private home charging availability will be the early adopters of electric mobility, as long as they need to use their EVs regularly and subsidies for EV purchase exist. Then, as EV market grows, publicly accessible EVSE with semi-fast charging capabilities are likely to appear in cities and traffic hotspots. In the meantime, technology development is expected to increase driving ranges while reducing costs, so that highway charging can also be profitable in the medium-term. Infrastructure deployment in the short term should be supported by favourable regulatory framework, as public charging beyond traffic hotspot is not profitable at current EV market growth rate. 


\section{References}

[1] Green eMotion project website. Project results available on-line at: http://www.greenemotionproject.eu/dissemination/deliverables.php

[2] Green eMotion, deliverable 9.4, "Envisaged EU mobility models, role of involved entities, and Cost Benefit Analysis in the context of the European Clearing House mechanism. Part 2". February 2015.

[3] German National Platform for Electromobility, "Fortschrittsbericht 2014 - Bilanz der Marktvorbereitung", December 2014.

[4] Green eMotion, deliverable 9.6, "Barriers, gaps, and commercial and regulatory framework for broad rollout of electromobility".

\section{Authors}
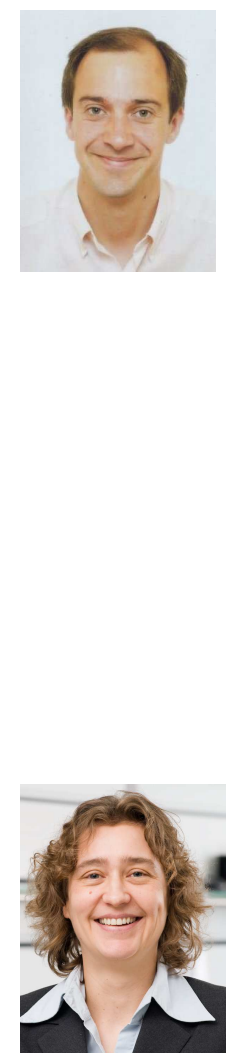

Carlos Madina is M. Sc. in Industrial Engineering (2001) from the School of Engineering of the University of the Basque Country in Bilbao. Since October 2001 to date, he has been working at the Energy Unit of TECNALIA, where he has participated in several private, national international research projects. He has knowledge about the regulations existing in different European countries and broad experience in developing and carrying out feasibility studies of innovative business models related to Distributed Energy Resources

Dr. Heike Barlag started her career at Siemens in 2002 in the Corporate Technology department, the Siemens research and development center. There she gained first experience in national and European research projects. Since 2010 she is working for the business unit Electric Vehicle Infrastructure as team leader for system technology and funded projects. She is in charge of Siemens activities in funded projects around electromobility - "Model Region Munich" and "EDISON" as well as "Crome" - and the development of a future proof technology for the Siemens EV infrastructure portfolio. Since the launch of the European Green eMotion project in March 2011, she is the responsible project coordinator of this electromobility demonstration project with 43 partners
Giovanni Coppola graduated in Electronics Engineering. $\mathrm{He}$ is currently in the Special Projects and Technological Development unit of Infrastructure and Networks Division, part of Enel SpA, Italy's largest power company, and Europe's second listed utility by installed capacity.

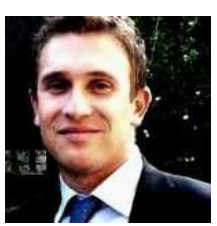
Besides leading the team effort in international $R \& D \& D$ projects, he is in charge of drawing out business models in the smart-grids field and managing the solutions development of smart-grids IT layers and alternative EV recharging infrastructures. Before joining Enel, he has worked as mixed-signal hardware engineer in semiconductors industry and academia, designing System-On-Chip products for energy efficiency and biomedical applications.

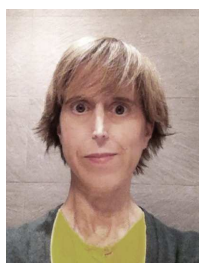

Inés Gómez, M. Sc. in Industrial Engineering (2002) from the School of Engineering of the University of the Basque Country in Bilbao. Since 2004 to date, at the Energy Unit of TECNALIA working in research projects mainly related to Distributed Energy Resources

Raúl Rodríguez, M. Sc. in Electrical Engineering (1996) from the School of Engineering of the University of the Basque Country in Bilbao. Since October 2000 to date, at the Energy Unit of LABEIN, now TECNALIA, working as researcher and project leader on socioeconomic and technical aspects of active distribution networks in the electrical system both in the frame of EU-funded and national research projects.

Dr. Eduardo Zabala received a $\mathrm{PhD}$ in Electronics Engineering in 1994 and a M. Sc. in Energy Engineering in 1984, both from the School of Engineering of the University of the Basque Country, Bilbao. 10 years' experience in electronics design and 5 years as EMC consultant and researcher. Now in charge of the EV Programme in TECNALIA Energy and Environment. Lecturer in the Engineering School of Bilbao since 1988. 